\title{
Zusammenfassung und Ausblick
}

In diesem Artikel wurde ein sozio-technischer Rahmen vorgestellt, mit dem Plattformanbieter drei Gestaltungsebenen der Digital Customer Experience analysieren und planen können. Die Spieleplattform Steam wurde mit der Fallstudienmethode untersucht, sodass Handlungsempfehlungen zur Gestaltung der Digital Customer Experience abgeleitet werden konnten. Die Anwendung der Handlungsempfehlungen wurden anschließend im Kontext von Automobilbranche, TV-Streaming Anbietern und anhand einer Plattform für Autoreparaturen beispielhaft diskutiert.

Die Ergebnisse der Arbeit richten sich an verantwortliche Entscheider im IT-Service-Development und IT-Service-Design, IT-Strategen und Business Architekten, die ihre Informationssysteme und digitalen Plattformen in Hinblick auf die Gestaltung der Digital Customer Experience entwickeln und bewerten wollen. Erstens zeigen die erarbeiteten inhaltlichen Erkenntnisse erprobte Möglichkeiten auf, die Digital Customer Experience erfolgreich zu gestalten. Sie folgen aus der Fallstudie und stellen eine gute Grundlage für die Übertragung in weitere Unternehmenskontexte dar. Zweitens liefern die vorgestellten Gestaltungsebenen einen Rahmen zur Strukturierung. Im Rahmen der Fallstudie hat sich zum Beispiel gezeigt, dass insbesondere die Gestaltung der sozialen Ebene, d. h. „Werte und Normen“, häufig vernachlässigt wird und größere Aufmerksamkeit erfordert. Das Modell kann die Analyse und Planung der eigenen Digital Customer Experience unterstuitzen, da es die Aufmerksamkeit des Entscheiders nicht nur auf service- und technologiebezogene Fragestellungen lenkt, sondern auch die Gestaltung digitaler sozialer Interaktionen hinterfragt. 